\title{
EXPERIMENTAL STUDY OF WEAK INTERACTIONS BY PRECISION MEASUREMENT OF RARE KAON DECAY
}

$\mathrm{DOE} / \mathrm{ER} / 40560--3 \mathrm{~B}$

DE92 016289

\section{PROGRESS REPORT}

For period Nov.1, 1991 -April 30, 1992

\author{
Roland Winston \\ The University of Chicago \\ 5640 S. Ellis Ave. \\ Chicago, IL 60637
}

\author{
April 1992 \\ Prepared for \\ The U.S. Department of Energy \\ Award no. DOE DE FG02 90ER 40560 Task B
}

\section{DISCLAIMER}

This report was prepared as an account of work sponsored by an agency of the United States Government. Neither the United States Government nor any agency thereof, nor any of their employees, makes any warranty, express or implied, or assumes any legal liability or responsibility for the accuracy, completeness, or usefulness of any information, apparatus, product, or process disclosed, or represents that its use would not infringe privately owned rights. Reference herein to any specific commercial product, process, or service by trade name, trademark, manufacturer, or otherwisw does not necessarily constitute or imply its endorsement, recommend' ' on, or favoring by the United States Government or any agency thereof. The views and opinions of authors expressed herein do not necessarily state or reflect those of the United States Government or any agency thereof.

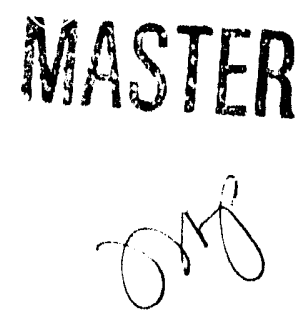




\section{PROGRESS REPORT}

Task B: Experimental Study of Weak interactions by Precision Measurement of Rare Kaon Decays

Table of Contents Dage

I. Overview 1

II. E731 Results 2

a. $\epsilon^{\prime} / \epsilon$ 3

b. $\Phi_{00}-\Phi_{+-}$

c. $\quad K_{L} \rightarrow \pi^{0} e^{+} e^{-}$

4

d. $\quad K_{L} \rightarrow \pi^{n} \gamma \gamma$

4

e. $\pi^{0} \rightarrow e^{+} e^{-}$

5

f. $\quad K L S \rightarrow \pi^{+} \pi^{-\gamma}$

6

g. $\mathrm{K}_{\mathrm{e4}}$ 7

h. $K_{e 3}$

7

i. $K_{L} \rightarrow 3 \pi^{0}$ decay constant $\varepsilon$

111. Fermilab E:773 Run 8

IV. Fermilab E799, Phase I 9

$\begin{array}{ll}\text { V. Publications } & 10\end{array}$

Appendix: Active Regenerator for E773 12 


\section{PROGRESS REPORT}

\section{OVERVIEW}

The long-term interest of this group is to carry out incisive and precise measurements which seek to clarify how hadrons interact weakly. At the present time this understanding can be summarized remarkably succinctly by the the Cabibbo-Kobayashi-Maskawa (CKM) parametrization. In the early 80's we were part of a collaboration that carried out the first precise measurement of Dolarized $\Sigma^{-} \beta$ decay (Fermilab Experiment \# 715). Our results (Publication 1) settled a long-standing experimental controversy as to the sign of $A / V$, the ratio of axial vector and vector form factors in this process. As is now well-known, the controversy was resolved in favor of the "standard model" CKM parametrization. But it is important to recall that this "A. $V$ test" was, at the time regarded as crucial to establishing the CKM pict'ure. In addition, we collected enough statistics to obtain a reasonably accurate measurement of "weak magnetism" and further, to hint that "weak electricity" which is firstorder forbidden, may in fact be larger than expected.

In 1984 we joined Bruce Winstein's NSF funded group studying decays of long-lived neutral kaons (Fermilab Experiment \# 731). The main physics thrust of $\mathrm{E} 731$ is to investigate the existence of direct $\mathrm{CP}$ violation in the $2 \pi$ decay modes of $K_{L}{ }_{L}$ by measuring the quantity Real $\left(\epsilon^{\prime} / \epsilon\right)$. Twenty-five years after the discovery of CP violation, its origin still remains a mystery. So far, CP violation has been observed in the two pion decays of the $\mathrm{K}^{0} \mathrm{~L}$ and in the charge asymmetry of its semileptonic decays. To the extent of our current knowledge, these instances of $\mathrm{CP}$ violation can be totally ascribed to the $\mathrm{CP}$ impurity in the weak interaction eigenstate $\mathrm{K}_{L}{ }_{L}$. Direct $\mathrm{CP}$ violation, i.e., $\mathrm{CP}$ change in the decay process rather than from the initial state impurity, appears to be rare, if it exists at all. The issue is far from resolved as there is an apparent discrepancy at approximately the two standard deviation level between this experiment, which finds no evidence for direct $\mathrm{CP}$ vislation, 
and a CERN experiment \# NA-31 which claims an effect. Clearly, more work is needed to settle the matter. Some clarification may emerge when we complete analysis of our entire data set, but it is becoming increasingly clear that a further generation of more precise $\epsilon^{\prime} / \epsilon$ experiments will be required.

Another way that direct $\mathrm{CP}$ violation could be observed is through the study of certain very rare $\mathrm{K}_{\mathrm{L}} \mathrm{L}$ decays. One such experiment, Fermilab E799, the search for $\mathrm{K}^{0} \mathrm{~L} \rightarrow \pi^{0} e^{+} e^{-}$, has collected phase I data during the 1991 fermilab fixed target run. This and other rare decay modes will be discussed subsequently.

\section{E731 RESULTS}

[In collaboration with Bruce Winstein's NSF funded group and Yau Wah (DOE Task J) at the University of Chicago, Elmhurst College, University of Illinois, Fermilab, and Saclay].

The principal physics goal of E731 is to investigate the existence of direct $\mathrm{CP}$ violation in the $2 \pi$ decay modes of $K_{L}$ by measuring the quantity Real $\left(\epsilon^{\prime} / \epsilon\right)$.

The incorporation of CP violation in the Standard Model is done via the CaDibbo-Kobayashi-Maskawa (CKM) mixing formalism. The CKM formalism ties together the two current unknowns of the Standard Model, namely, the toP quark mass and the magnitude of the direct $C P$ violation. The Standard Model requires the existence of direct CP violation, but depending on the top quark mass, its magnitude can be quite small. Thus, a def initive measurement of the $\epsilon^{\prime} / \epsilon$ parameter along with a measurement of the top quark mass would provide a very powerful check of the standard Model. A significant inconsistency between the values (or limits) of these two parameters would signal a problem with the standard Model and would be very interesting indeed. 
The current status of the $\epsilon^{\prime} / \epsilon$ measurement is as follows. In 1988, the CERN-NA31. group published a 3 standard deviation non-zero measurement of $\epsilon^{\prime} / \epsilon\left[(+33 \pm 11) \times 10^{-4}\right]$. In 1991, our group published a lower value that is consistent with zero value $\left[(-3 \pm 14 \pm 6) \times 10^{-4}\right]$ based upon $20 \%$ of the E731 data (Publication 2). Ours was the first determination in which all four $K_{L, S} \rightarrow \pi \pi$ decay modes were recorded simultaneously, allowing us to achieve the smallest systematic error $\left( \pm 6 \times 10^{-4}\right)$. Since then, the NA31 group has announced results from their 1988 and 1989 data, and still report a three standard deviation effect $\left[(23 \pm 3.4 \pm 6.5) \times 10^{-4}\right]$.

Also, as described below, our group has announced a preliminary result of $(+6 \pm 6.1 \pm 3.2) \times 10^{-4}$ from $100 \%$ data sample of the E731 data, which is still consistent with zero, and hence with the superweak model. The NA-3l central value lies somewhat above the current standard Model prediction, whereas our value is corisistent with current Standard Model prejudices at the lower end. At this point, the only safe conclusion that one can draw is that more precise measurements as well as further improvements in the theoretical framework for predicting $\epsilon^{\prime} / \epsilon$ are needed. We have also extracted a number of other important physics results from our E731 data. These will be mentioned briefly.

(a) $\epsilon^{\prime} / \epsilon$

We completed data taking on E-731 on February 15, 1988. The run was highly successful in terms of apparatus and accelerator performance. Our entire data set (some 5,000 tapes) contains approximately 300,000 $\mathrm{K}^{0} \mathrm{~L} \rightarrow 2 \pi^{0}$ events. We first reduced $20 \%$ of our total data by July 1988 , and subsequently published the $\epsilon^{\prime} / \epsilon$ result from it as mentioned above. Over the last two years, the E731 group has reduced the entire data sample, successfully understood the characteristics of the apparatus and the data, and announced a preliminary result of $(+6 \pm 6.1 \pm 3.2) \times 10^{-4}$ for the entire data set. Currently, we are in the process of finalizing this result, and somewhat improving it by extending the decay region, and 
further understanding the systematics.

A substantial part of this analysis, specially for the high statistics $\mathrm{K}_{\mathrm{e}} 3$ and $3 \pi 0$ modes, has been carried out on a 30-node, 2 M-byte ACP I system based at chicago.

b) CPT violating phase difference $\phi_{00}-\phi_{+-}$

From the same $20 \%$ of the E731 data, our group has published a new measurement of the CPT violating phase difference $\triangle \Phi=\phi_{00}-\phi_{+-}$ (Publication 3). The result is $\triangle \Phi=-0.9^{0} \pm 2.2^{0} \pm 0.7^{0}$. No evidence for CPT violation was found. This result was significant in light of the earlier measurement, $12^{0} \pm 6^{\circ}$, a two standard deviation departure. We have announced a preliminary result of from our 100\% data sample, and expect. to finalize it soon. Our group has also run Fermilab experiment 773 with the goal of measuring this phase difference to half a degree.

(c) $K_{L} \rightarrow \pi^{0} e^{+} e^{-}$

The amplitude for $K_{L} \rightarrow \pi^{0} e^{+} e$ is composed of a CP conserving part and a CP violating part. The leading $\mathrm{CP}$-conserving amplitude proceeds through two-photon exchange while the CP-violating one may proceed via one-photon exchange. Within the framework of the Standard Model where $C P$ violation comes from the phase $\delta$ in the Kobayashi-Maskawa matrix. $K_{L} \rightarrow \pi^{0} e^{+} e^{-}$may have a sizable $\Delta S=1 C P$-violating component $\left(\epsilon^{\prime} \pi e e^{/ \epsilon}\right)$.

In contrast, the Superweak Model predicts $\epsilon^{\prime}$ mee to be zero. In terms of the CP eigenstates $K_{1}$ (even) and $K_{2}(O d d), K_{L}$ is written as $\left(K_{2}+\epsilon K_{1}\right)$ and one may describe the contributions to the $K_{L} \rightarrow \pi^{0} e^{+} e^{-}$ decay amplitude as follows: (a) the $\mathrm{CP}$ conserving amplitude via two photons exchange $K_{2}->\pi \gamma^{*} \gamma^{*}$, where $\gamma^{*}$ is an off-shell photon; (b) the 
mass matrix "indirect" $C P$ violation amplitude which comes from the small $K_{1}$ impurity in $K_{L}$; and $(C)$ the "indirect" CP violation amplitude $\epsilon^{\prime}$ from the one photon exchange $K_{2}->\pi^{0} \gamma^{*}$ part of $K_{L}$. Within the standard Model, part ( $c$ ) can be described by the "electromagnetic penguin" diagram and is comparable to or larger than part (b) in magnitude. Theoretical estimation of the "size" of this penguin is under active pursuit by our theoretical colleagues. While the predictions of the branching ratio in general are around $10^{-11}$, the Weinberg Model predicts a branching ratio as high as $0.6 \times 10^{-9}$. The $K_{L} \rightarrow>\pi^{0} e^{+} e^{-}$decay is an attractive alternative avenue for the observation of 'direct' $C P$ violation, particularly if the studies of the $2 \pi$ decays of neutral kaon $\left(\epsilon^{\prime} / \epsilon\right)$ should prove inconclusive.

A limit of B.R. $\left(K_{L}->\pi^{0} e^{+} e^{-)}<7.5 \times 10^{-9}\right.$ (90\% C.L.) has been obtained from the complete E-731 data set [Publication 4]. This is in agreement with the BNL E-845 result, B.R. $\left(K_{L} \rightarrow \pi^{0} e^{+} e^{-}\right)<5.0 \times 10^{-9}$ (90\% C.L.). E-799 at Fermilab, an experiment dedicated to search for this decay mode has taken data in its preliminary run during the 1991 Fermilab fixed target run. There is also keen competititon from KEK.

(d) $K L \rightarrow \pi^{0} \gamma \gamma$

$\mathrm{KL} \rightarrow \pi^{0} \gamma \gamma$ is of current interest for several reasons. It provides an opportunity to test the standard Model in the context of chiral symmetry: at the one-100p level in chiral perturbation theory, the decay has a predicted branching ratio of $6.8 \times 10^{-7}$ with a characteristic $\gamma \gamma$ mass distribution having a peak at about $325 \mathrm{MeV}^{2}$. This decay also provides a $\mathrm{CP}$ conserving intermediate state for the $\mathrm{KL} \rightarrow \pi^{0} e+e$-decay. One needs to measure $\mathrm{KL} \rightarrow \pi^{0} \gamma \gamma$ well so that the interpretation of direct $\mathrm{CP}$ violation in $\mathrm{KL} \rightarrow \pi^{0} e+e-c a n$ be unambiguous. For our $20 \%$ data analysis, a result of B.R. $\left(\mathrm{KL} \rightarrow \pi^{0} \gamma \gamma\right)<2.7 \times 10^{-6}$ (90\% C.L.) was published in 1989 (Publication 5). Our current $100 \%$ data analysis

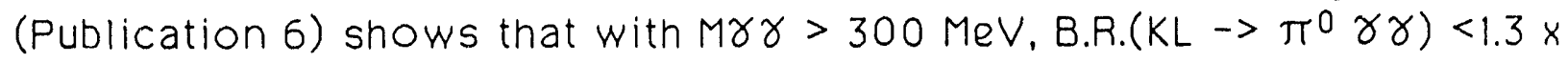

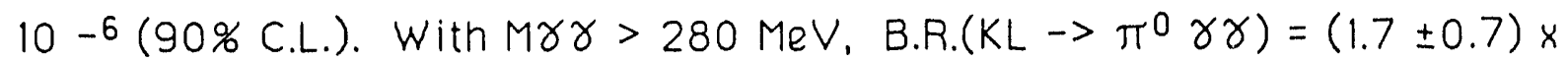


10-6. This is in agreement with the CERN NA31 result,B.R. $\left(K L \rightarrow \pi^{0} \gamma \gamma^{\prime}\right)=$ $(2.1 \pm .6) \times 10^{-6}$. for the same mass region. This was the subject of a Ph.D. thesis by $V$. Papadimitriou.

(e) $\pi^{0} \rightarrow e^{+} e^{-}$

Basically this decay can be described by a fourth order electromagnetic diagram and the branching ratio is calculated to be > $4.8 \times 10^{-8}$ (the unitarity limit). The world average B.R. $\left(\pi^{0}>e^{+} e\right)=(1.8 \pm$ $0.7) \times 10^{-7}$ comes from two measurements, but there is some controversy concerning the validity of the results. A new technique was used to search for this decay in the current $E-731$ data. We have collected about 15 million reconstructed $K \rightarrow 3 \pi^{0}$ decays. The signature of $\pi^{0->e^{+}} e^{-}$is then two tracks matched with leadglass showers and reconstructed as a $\pi^{0}$, the four extra showers reconstructed as $2 \pi^{0}$, and all six clusters reconstructed as a kaon coming from the target. The total sample corresponds to a limit of $<2.0 \times 10^{-7}$ ( $90 \%$ confidence). There is no background at this level. We believe this "tagged pion" technique can be exploited much further and a definite measurement can be made in the upcoming E799.

(f) $K_{L, S} \rightarrow \pi^{+} \pi^{-} \gamma$

The radiative decay of $K_{L, S}{ }^{->} \pi^{+} \pi^{-} \gamma$ can originate from (a) inner bremsstrahlung from the virtual pions and (b) direct emission from the $K \pi \pi$ vertex. The inner bremsstrahlung is common to both $K_{L}$ and $K_{S}$ decays with a CP-even final state. For decays arising from direct emission the final state is $\mathrm{CP}$-odd, and therefore allowed only in the $\mathrm{K}_{\mathrm{L}}$ and suppressed in $K_{S}$ decays. The $2 \pi$ radiative events were selected by requiring two charged tracks and one photon shower in the leadglass calorimeter. The $\mathrm{K}_{\mathrm{S}}$ decays has the characteristic $1 / \mathrm{k}$ spectrum of inner bremsstrahlung. The 
$K_{L}$ decays have both the $1 / k$ and the $C P$-allowed direct emission.

Results for these $2 \pi$ radiative decays from E731 are:

B.R. $\left(K_{S} \rightarrow \pi^{+} \pi^{-} \gamma, E_{\gamma}>50 \mathrm{MeV}\right)=(2.41 \pm 0.06) \times 10^{-3} \times$ B.R $\left(K_{S} \rightarrow \pi^{+} \pi^{-}\right)$,

B.R. $\left(K_{L}, \rightarrow \pi^{+} \pi^{-} \gamma, E_{\gamma}>20 \mathrm{MeV}\right)=(1.45 \pm 0.09) \times 10^{-5}$.

These represent major improvements from previous results. Also from the $\mathrm{K}_{\mathrm{S}}$ decays, one can extract from the proper time distribution behind the regenerator, the parameter $\eta_{+-\gamma} \gamma$. It is measured to be $\eta_{+-} \gamma=$ $(2.0 \pm 0.2) \times 10^{-3}$. This is the 4 th unambiguously non-zero CP violation parameter ever measured joining $\eta_{+-}, \eta_{00}$ and $\delta$.

(g) $\mathrm{K}_{e_{4}}$

Before this experiment, only 15 events had been observed. We have a total of 780 events observed yielding a branching ratio of B.R. $\left(K_{L} \rightarrow \pi^{ \pm} \pi^{0} e^{ \pm} v\right)=(5.6 \pm 0.2 \pm 0.4) \times 10^{-5}$. This decay mode, unlike its charged partner $\mathrm{K}^{+} e^{4}$ is $\mathrm{P}$-wave dominated rather than $\mathrm{s}$-wave. Further angular distribution analysis may also test various form factor contributions. One of them could be direct $C P$ violatec.

(h) $k_{e 3}$

Since the $\epsilon^{\prime} / \epsilon$ measurement requires a very thorough understanding of the detector acceptance, we collected approximately 100 million $\mathrm{Ke}_{3}$ events during the E731 run in order to monitor the charged mode acceptance. This is the largest sample of $\mathrm{Ke}_{3}$ events ever collected and not surprisingly, is an avenue for independent physics measurements. We expect to measure the $\mathrm{CP}$ violating semi-leptonic asymmetry parameter $\delta$ to a precision of $\pm 0.008 \%$; the current world average has a precision of $\pm 0.012 \%$. These data may also yield a new measurement of the $\mathrm{Ke}_{3}$ form 
factor.

(i) $K_{L} \rightarrow 3 \pi^{0}$ decay consiant

In a similar fashion, we wrote appruximately 15 million $K_{L} \rightarrow 3 \pi^{3}$ decays to tape during E73! to monitor the neutral mode acceptance of the detector. Again, this is the largest $3 \pi^{0}$ sample to date. We have published the first-ever measurement of the quadratic slope term in the $3 \pi^{0}$ Dalitz plot [Publication 7]. Our value is $(-0.33 \pm .11 \pm .07) \%$. This quantity is closely tied to the second order term in the Chiral Lagrangian, and can be used to confront the Lattice Gauge calculations. The $3 \pi^{0}$ mode offers a very clean way of measuring the quadratic slope term of the Dalitz plot since there are no charged particles in the final state. Also, because of the three-fold symmetry. only the $3 \pi^{0}$ decay has an identically zero linear slope term, and as a result, the quadratic term has no corrections. For a decay such as $K_{L} \rightarrow \pi^{+} \pi^{-} \pi^{0}$, the quadratic slope term is dominated by the square of the linear term.

III. FERMILAB E- 773 RUN.

Neutral Kaons offer one of the most sensitive ways of investigating the possibility of CPT violation in nature. Assuming CPT symmetry, our current experimental knowledge of the phase and magnitude of $\epsilon$, the values of the strong $\pi \pi$ phase shifts, and the ratio $\eta_{00} / \eta_{+-}$dictate that the phase difference $\Delta \Phi$ between $\eta_{00}$ and $\eta_{+-}$must be less than a fraction of a degree. A value of $\triangle \Phi$ larger than this would clearly signal a violation of CPT invariance and its measurement would occasion considerable excitement. This experiment took excellent data during the 1991 fixed target run at Fermilab, and should be able to determine $\Delta \Phi$ to an accuracy of half a degree. The data set has already been reduced and separated within a record time of 3 months after end of the 1991 fixed target run. 
The apparatus for E773 consists of the E731 spectrometer with appropriate modifications. These modifications consisted of changes in the beamline, a new configuration with two regenerators instead of one, and improvement in the charged trigger.

A major new feature of the experiment is the presence of a fully active downstream regenerator which moves periodically between the two Kaon beams. Unlike its E731 counterpart, the new regenerator is made entirely out of scintillator material instrumented with photomultiplier tubes. This feature allows one to study and reject the $\mathrm{K}_{S}$ events arising from non-coherent regeneration. It also allows the rejection of events that produce cross-over between the two kaon beams, thus reducing the systematic error. Due to a very high hadronic shower background rate, observing the very small signals resulting from diffractive regeneration in the scintillator is a challenging task. In addition, the signals have to be delayed significantly prior to digitization in order to be in time with the rest of the detector. The active regenerator overcame these challenges by employing fast scintillator and photomultiplier tubes, active voltage dividers and shaping amplifiers. A more detailed description of the active regenerator is given in Appendix A.

The regenerator performed very well during the E773 data run. It was able to tolerate the high rates produced by up to $2 \times 10^{12}$ protons per pulse without excessive PMT anode current. We find that the coherent peak $\left(p_{t}{ }^{2}=0\right)$ is reduced by only $2 \%$ due to accidental loss, whereas the background events are reduced by an enormous 68\%.

IV. FERMILAB E-799, PHASE I

E799 which has an ultimate goal of observing $K_{L} \rightarrow \pi^{0} e^{+} e^{-}$with a sensitivity of $<1 \times 10^{-11}$ ( $90 \%$ C.L.), was approved by the Fermilab PAC in June 1989 for two fixed target runs. The experiment ran for two months 
during the 1991 fixed target run in Fermilab, with three transition radiation detectors installed. Besides the $K_{L} \rightarrow \pi^{0} e^{+} e^{-}$mode, the data should be sensitive to a host of other rare decays.

V. Publications

1. High-precision measurement of polarized- $\Sigma^{-}$beta decay, S.Y. Hsueh, D. Muller, J. Tang, R. Winston, G. Zapalac, E.C. Swallow, et al., Phys. Rev. D. $38,2056,(1988)$.

2. Determination of $\operatorname{Re}\left(\epsilon^{\prime} / \epsilon\right)$ by the simultaneous detection of the four $K_{L, S} \rightarrow \pi \pi$ decay modes, J.R. Patterson, A. Barker, R.A. Briere, L.K. Gibbons, G. Makoff, V. Papadimitriou, S. Somalwar, Y.W. Wah, B. Wiknstein, R. Winston, M. Woods, H. Yamamoto, E. Swallow, et al., Phys. Rev. Lett. 64, 1491, (1990).

3. A test of CPT symmetry through a determination of the difference in the phases of $\eta_{00}$ and $\eta_{+-}$in $K->2 \pi$ decays, M. Karlsson, G. Gollin, J. Okamitsu, R. Tschirhart, A. Barker, R.A. Briere, L.K. Gibbons, G. Makoff, V. Papadimitriou, J.R. Patterson, S. Somalwar, Y.W. Wah, B. Winstein, R. Winston, M.Woods, H. Yamamoto, E. Swallow, et al., Phys. Rev. Lett 64 , 2976 (1990).

4. New limit on $K_{L} \rightarrow \pi^{0} e^{+} e^{-}$. A. Barker, R. Briere, L.K. Gibbons, $G$. Makoff, V. Papadimitrious, J.R. Patterson, S. Somalwar, Y.W. Wah, B. Winstein, R. Winston, M. Woods, H. Yamamoto, E. C. Swallow, et al., Phys. ReV. D. 41, 3546, (1990).

5. Search for $K_{L} \rightarrow \pi^{0} \gamma \gamma, V$. Papadimitriou, L.K. Gibbons, J.R.

Patterson, Y. Wah, B. Winstein, R. Winston, M. Woods, H. Yamamoto, E.C. Swallow, et al., Phys. Rev. Lett. 63, 28, (1989). 
6. A measurement of the branching ratio of the decay $K_{L} \rightarrow \pi^{0} \gamma \gamma$, V. Papadimitriou, A.Barker,R.A.Briere,L.K. Gibbons, G.Makoff,J.R. Patterson, S.Somalwar, Y. W.Wah, B. Winstein, R. Winston, M. Woods, H. Yamamoto, E.C. Swallow, et al., Phys. Rev. D44, 573, (1991).

7. A measurement of the quadratic slope parameter in the $K_{L} \rightarrow 3 \pi^{0}$ decay dalitz plot, S.V.Somalwar, A.Barker,R.A.Briere.L.K. Gibbons, G.Makoff, V.Papadimitriou,J.R. Patterson,Y. W.Wah, B. Winstein, F. Winston, H. Yamamoto, E.C. Swallow, et al., Phys. Rev. Lett. 1Z, 2580, (1992).

8. Search for the decay $K_{L}>\pi^{0} v v, G . E$. Graham, A. Barker, R. A. Briere, L.K. Gibbons, G. Makoff, V. Papadimitriou, J.R. Patterson, S. Somalwar, Y.W. Wah, B. Winstein, R. Winston, H. Yamamoto, E.C. Swallow, et al., Submitted to Phys. Rev. Lett., 20 April 1992. 


\section{APPENDIX}

\section{ACTIVE REGENERATOR FOR E773}

The active regenerator consists of a total of 0.4 meters ofplastic scintillator in the Kaon beamline. Its purpose is to regenerate a $\mathrm{K}_{\mathrm{S}}$ heam from the $K_{L}$ beam. The scintillator is viewed by photomultiplier tubes in order to distinguish the coherent regeneration from the ineleastic and diffractive regeneration. Since the presence of a signal in the PMT is caused by a localized interaction, such a signal implies non-coherent regeneration. The length of the regenerator was decided on the basis of an optimization study of the E773 experiment.

The background light in the scintillator is produced mostly by the charged particles produced in hadronic showers. Due to the high rate of hadronic showers, pulse pile-up is expected to be a problem, especially since the signal size from a typical diffractive event can be as small as a few photoelectrons. On the other hand a hadronic shower can create as many as 20,000 photoelectrons in a " thick scintillator. If the shower occurs in the accelerator rf bucket previous to the one in which the signal occurs, the PMT has to recover completely in approximately 20 nsec. In addition, the PMT output has to be delayed by approximately 500 nsec to give the trigger enough time to make the digitizing decision. The dispersion in the delay cable exacerbates the signal pile-up.

A possible solution is to have individual scintillator blocks much smaller than 1", which is clearly impractical. The proposed solution to avoid the pile-up is to use fast scintillator in conjunction with fast PMT's, and shape the tube output with a high-pass filter in order to eliminate the slow components entirely. The price one pays is in the attenuation of the signals. This attenuation is in addition to the attenuation due to the delay cable. Because of the average anode current limitation $(<100 \mu \mathrm{A})$, one cannot increase the PMT gain enough to compensate for the attenuation due to the delay cable and shaping circuit. As a result, one has to resort to low-noise external amplification of the PMT signal. 
One additional problem encountered in a high rate PMT environment is that of random after pulsing due to the residual yas in the PMT. The amount of after pulsing depends on the total PMT activity. which, as stated above, is very large in our case. As a result, the probability of seeing a random afterpulse of expected signal size in at least one of the channels of the detector is significant. For example, with the expected hadronic shower background in E773, one would, on an average, get aftorpulses bigger than ten photoelectrons in at least one of the channels in the regenerator once in 5 pulses. The Carbon diffractive signal is then simpiy washed out. The after pulsing probability is eliminated to a large degree by having more than one PMT look at the same scintillator, and demanding multiple coincidence.

Finally, che also needs excellert optical collection in order to see the very small signals that one expects from diffractive events. The PMT is mounted directly on the scintillator. Teflon tape normally used as a pipe sealant works as an efficient diffuse reflector for wrapping the scintillator.

The regenerator relies very heavily on the electronics to overcome the severe environmental conditions described above. To this end, we designed and custom-made the amplifiers for the PMT's.

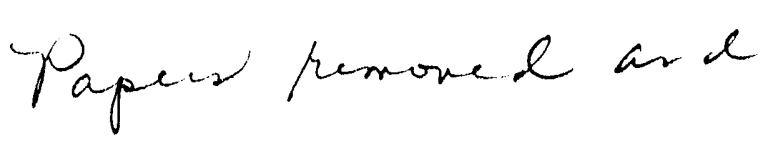<smiles>[Ca][Ca][Te]</smiles>

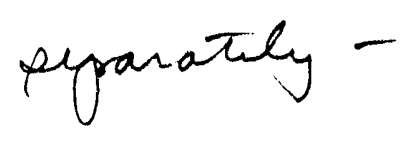



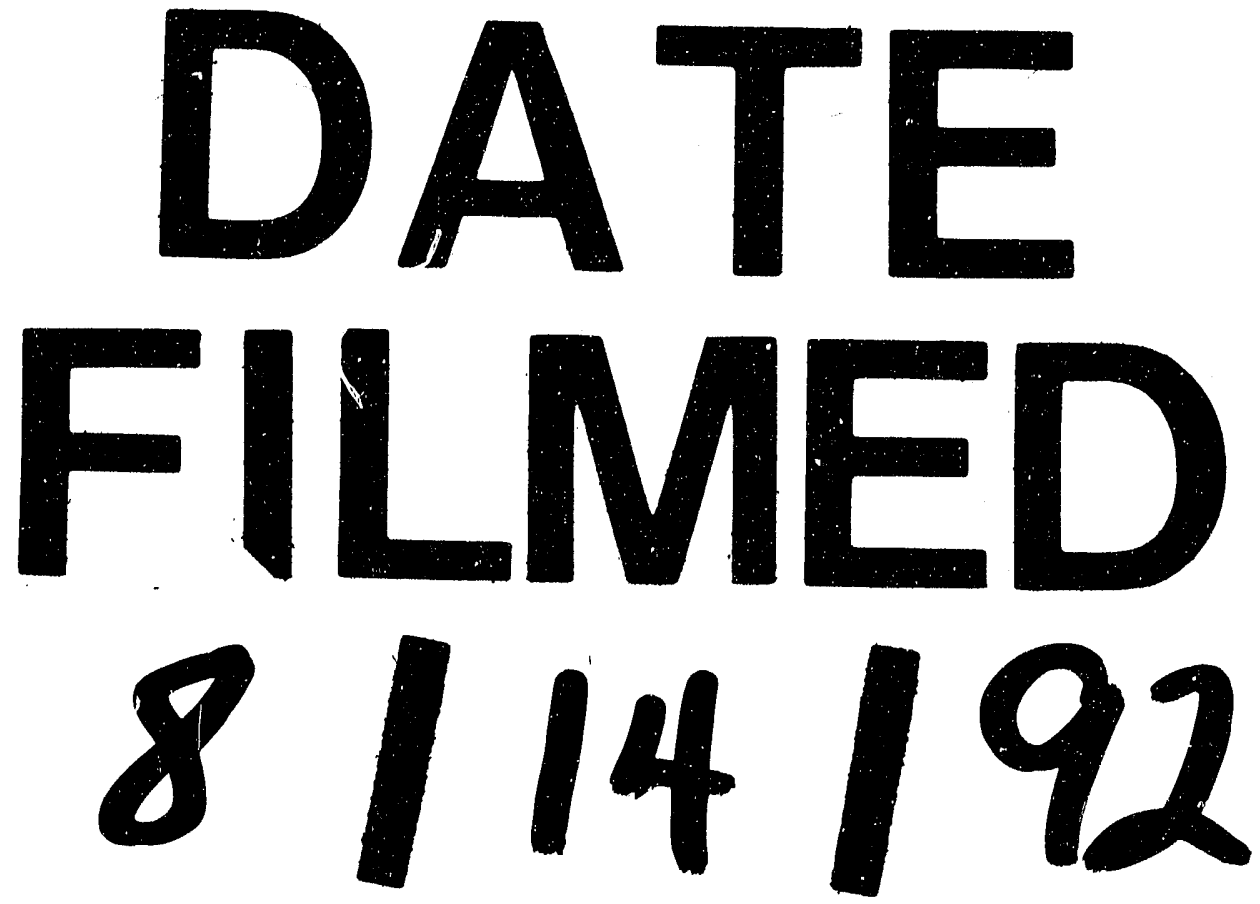
